\title{
Asymptotic solution of mode-III crack in damaged softening materials
}

\author{
X.T. ZHANG ${ }^{1}$, K.C. HWANG ${ }^{2}$ and T.H. HAO \\ ${ }^{1}$ Institute of Mechanics, Chinese Academy of Sciences, Beijing 100080. China; \\ ${ }^{2}$ Department of Engineering Mechanics, Tsinghau University, Beijing 100084, China; \\ ${ }^{3}$ Department of Basics, China Textile University, Shangai 20005I, China.
}

Received 7 November 1992; accepted in revised form 26 April 1993

\begin{abstract}
In this paper, a constitutive model of elasticity coupled with damage suggested by Lemaitre et al. [1] is used. The macroscopic stress-strain response of the model includes two stages: strain hardening and strain softening. The basic equation is derived for the anti-plane shear problem. Several lowest order asymptotic solutions are obtained, and assembled for the crack-tip fields.
\end{abstract}

\section{Introduction}

In fracture mechanics it is usually assumed that the materials both nearby and far away from the crack tip are non-damaged. In fact strain concentration near the crack tip always leads to nucleation, growth and coalescence of microcracks or microvoids. Consequently, the constitutive behavior of the material near the crack tip becomes significantly different from that of the material far away from the crack tip. Usually, the damage leads to weaker singularity of the crack-tip field. Taking account of damage in the constitutive law can better reflect the act ial behavior of materials.

In the last decade, although a lot of progress has been made in the investigation -f fracture associated with damage, few solutions are available on the crack-tip field in di.naged materials. Bui et al. [2] studied the dynamic propagation of mode-III crack in elastic and elasto-plastic damaged medium and obtained the closed form solutions. A finite element solution was obtained for mode-I crack. Chow et al. [3] proposed an anisotropic damage model. The model was next applied to investigate the crack-tip shielding effect due to microcracking, and a closed form asymptotic solution for a stationary crack under plane strain mode-I loading was obtained. Recently, Wang and Chow [4] investigated th? effects of the distributed damage in terms of the damage variable $D$ on the HRR field. I the materials in both [3] and [4] are not softening. Hao, Zhang and Hwang [i] (ii. :after denoted by $\mathrm{HZH}$ ) analytically obtained the anti-plane shear field of a stationary crack for the elasto-brittle damaged material under the condition of small scale damage. In the present paper the near-tip fields for mode-III crack in a class of elasto-damaged material is obtained. The adopted constitutive relation can reflect the so-called post-peak strain softening behavior which appears usually in deformation process of non-metals, such as concrete. 


\section{Constitutive relations}

Following Lemaitre et al. [1] the constitutive relations for an isotropic elastic material with isotropic damage are as follows

$$
\sigma_{i j}=2 \mu(1-D)\left(\varepsilon_{i j}+\frac{v}{1-2 v} \varepsilon_{k k} \delta_{i j}\right)
$$

where $\mu$ is the elastic shear modulus, $v$ the Poisson's ratio and $D$ the damage parameter. For the convenience of investigating the anti-plane problem, we define the equivalent shear strain and equivalent shear stress as

$$
\begin{aligned}
& \varepsilon_{e q}=\left(\frac{1}{2} \varepsilon_{i j}^{\prime} \varepsilon_{i j}^{\prime}\right)^{1 / 2}, \\
& \tau_{e q}=\left(\frac{1}{2} \sigma_{i j}^{\prime} \sigma_{i j}^{\prime}\right)^{1 / 2},
\end{aligned}
$$

where $\varepsilon_{i j}^{\prime}$ and $\sigma_{i j}^{\prime}$ are deviatoric tensors of strain and stress, respectively. Following [1], the damage evolution law is taken as

$$
\mathrm{d} D=\left\{\begin{array}{l}
\left(\varepsilon_{e q} / \varepsilon_{0}\right)^{s} \mathrm{~d} \varepsilon_{e q}, \quad \text { when } \varepsilon_{e q}=\varepsilon_{D} \text { and } \mathrm{d} \varepsilon_{e q}=\mathrm{d} \varepsilon_{D} \geqslant 0 \\
0, \text { when } \varepsilon_{e q}<\varepsilon_{D} \text { or } \mathrm{d} \varepsilon_{e q}<0
\end{array}\right.
$$

where $s$ is a material parameter, $\varepsilon_{D}$ is the maximum of $\varepsilon_{e q}$ in the deformation history of the material, $\varepsilon_{0}$ a reference strain which will be related to the rupture strain of the material. Irreversibility of damage requires $\mathrm{d} D \geqslant 0$. For the case of active loading ( $\mathrm{d} \varepsilon_{e q} \geqslant 0$ ) from the virginal state $D=\varepsilon_{D}=0$, the integration of (2.4) gives

$$
D=\left(\varepsilon_{e q} / \varepsilon_{R}\right)^{s+1}
$$

where $\varepsilon_{R}=\left[(s+1) \varepsilon_{0}^{s}\right]^{1 /(s+1)}$ is the shear rupture strain of the material. It is natural to assume

$$
s+1>0
$$

The relation between $\tau_{e q}$ and $\varepsilon_{e q}$ can be obtained from (2.1)-(2.5)

$$
\tau_{e q}=2 \mu\left[1-\left(\varepsilon_{e q} / \varepsilon_{R}\right)^{s+1}\right] \varepsilon_{e q} .
$$

Noticing (2.5) and (2.7), if $\varepsilon_{e q}=\varepsilon_{R}$, we have $D=1, \tau_{e q}=0$, the materials are fully damaged and cannot sustain any load. The $\tau_{e q} \sim \varepsilon_{e q}$ curves described by (2.7) are shown in Fig. 1. There is a maximum of $\tau_{e q}$ for every curve which is denoted by $\tau_{e q}^{*}$. The value of $\varepsilon_{e q}$ and $D$ corresponding to $\tau_{e q}^{*}$ are denoted by $\varepsilon_{e q}^{*}$ and $D^{*}$, respectively. And we have from (2.7)

$$
D^{*}=\left(\varepsilon_{e q}^{*} / \varepsilon_{R}\right)^{s+1}=1 /(s+2) .
$$




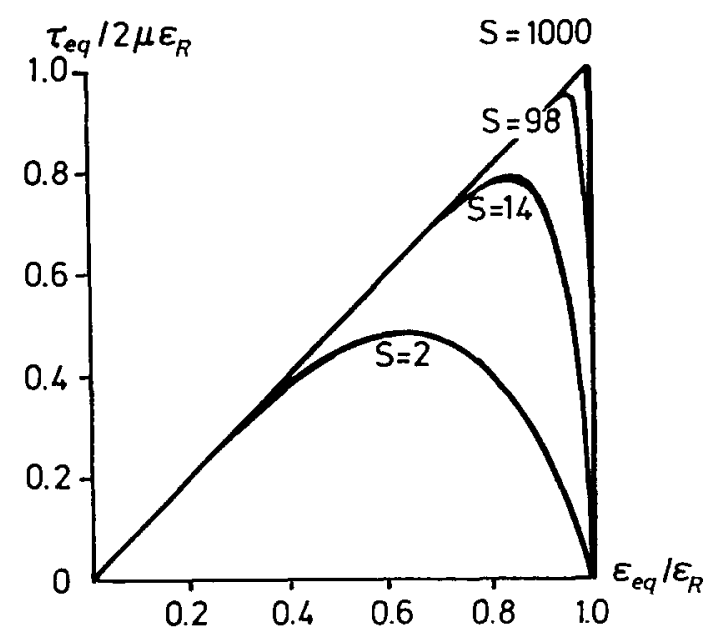

Fig. 1. Equivalent shear stress vs. equivalent shear strain.

The domains of $D<D^{*}$ and $D>D^{*}$ can be called hardening and softening zone, respectively. The domain of $D=1$ is called fully damaged zone. Letting $s \rightarrow \infty$, the material shown in Fig. 1 becomes the elasto-brittle damaged material considered by $\mathrm{HZH} \mathrm{[5].}$

\section{Basic equation and its asymptotic solutions}

Utilizing cylindrical coordinates $(r, \theta)$ centered at the crack tip, (2.1) becomes for the anti-plane problem

$$
\tau_{\alpha}=\mu(1-D) \gamma_{\alpha}, \quad \alpha=r, \theta,
$$

where $\tau_{\alpha}=\sigma_{\alpha z}, \gamma_{\alpha}=2 \varepsilon_{\alpha z}$. The equation of equilibrium is

$$
\frac{\partial \tau_{r}}{\partial r}+\frac{\tau_{r}}{r}+\frac{1}{r} \frac{\partial \tau_{\theta}}{\partial \theta}=0
$$

The geometric relations for small deformation are

$$
\gamma_{r}=\frac{\partial w}{\partial r}, \quad \gamma_{\theta}=\frac{1}{r} \frac{\partial w}{\partial \theta}
$$

where $w$ is out-of-plane displacement. Associating (3.1), (3.2) with (3.3), the basic equation is obtained

$$
(1-D) \nabla^{2} w-\tilde{\nabla} w \cdot \tilde{\nabla} D=0,
$$

where $\tilde{\nabla}$ and $\nabla^{2}=\tilde{\nabla} \cdot \tilde{\nabla}$ are gradient and Laplacian operator, respectively. Making use of (3.3), (2.2) and (2.5) the damage parameter $D$ can be denoted as

$$
D=\left\{\frac{1}{2 \varepsilon_{R}}\left[\left(\frac{\partial w}{\partial r}\right)^{2}+\left(\frac{1}{r} \frac{\partial w}{\partial \theta}\right)^{2}\right]^{1 / 2}\right\}^{s+1} .
$$


Assume that the leading term of the asymptotic expansion of the displacement field has the form with variables separable

$$
w / 2 \varepsilon_{R}=w_{0}+r^{q} F(\theta)+\{\text { higher order terms in } r\}
$$

where $w_{0}=$ const. is the shear opening displacement at the crack tip. When the fully damaged zone appears ahead of the crack, $w_{0} \neq 0$ is possible. Obviously $w_{0}$ makes no contribution to the strains. In (3.6) $q$ is the singularity exponent of the leading term of the displacement field. For the strain to be bounded, $q \geqslant 1$ is required. The value of $q$ will be determined or discussed according to the different cases. For the sake of simplicity, denote $F(\theta), \mathrm{d} F / \mathrm{d} \theta$ and $\mathrm{d}^{2} F / \mathrm{d} \theta^{2}$ by $F, F^{\prime}$ and $F^{\prime \prime}$. Substituting (3.6) into (3.5), we have

$$
D=r^{(q-1)(s+1)}\left(q^{2} F^{2}+F^{2}\right)^{(s+1) / 2}+\{\text { higher order terms in } r\} .
$$

Making use of (3.6) and (3.7), Eqn. (3.4) can be rewritten in the order of the power of variable $r$ as follows

$$
\begin{aligned}
& r^{p_{1}}\left(q F+F^{\prime \prime}\right)-r^{p_{2}}\left[\left(q^{2} F+F^{\prime \prime}\right)\left(q^{2} F^{2}+F^{\prime 2}\right)^{(s+1) / 2}+q(q-1)(s+1) F\left(q^{2} F^{2}+F^{\prime 2}\right)^{(s+1) / 2}+\right. \\
& \left.+(s+1) F^{\prime 2}\left(q^{2} F+F^{\prime \prime}\right)\left(q^{2} F^{2}+F^{\prime 2}\right)^{(s-1) / 2}\right]+\{\text { higher order terms in } r\}=0,
\end{aligned}
$$

where the notations are introduced for abbreviation

$$
\left.\begin{array}{l}
p_{1}=q-2 \\
p_{2}=(s+2) q-(s+3)
\end{array}\right\} .
$$

In (3.8), the 1st and 2nd parts represent the contributions of elasticity and damage to the basic equation, respectively. We distinguish different cases of the relation between $p_{1}$ and $p_{2}$, and retain hereafter only the leading term in all equations.

\subsection{Case $p_{1}=p_{2}$.}

The elasticity and damage terms are equally dominant and from (3.9) $q=1$. Equation (3.8) reduces to

$$
\left(F+F^{\prime \prime}\right)\left\{\left(F^{2}+F^{\prime 2}\right)^{(s-1) / 2}\left[F^{2}+(s+2) F^{\prime 2}\right]-1\right\}=0,
$$

which can be split into two equations

$$
\begin{aligned}
& F+F^{\prime \prime}=0, \\
& \left(F^{2}+{F^{\prime}}^{2}\right)^{(s-1) / 2}\left[F^{2}+(s+2) F^{2}\right]=1 .
\end{aligned}
$$

Differentiating (3.11) against $\theta$, we get

$$
\begin{aligned}
& F^{\prime}\left(F^{2}+F^{\prime 2}\right)^{(s-3) / 2}\left\{(s-1)\left(F+F^{\prime \prime}\right)\left[F^{2}+(s+2) F^{\prime 2}\right]+\right. \\
& \left.\quad+2\left(F^{2}+F^{\prime 2}\right)\left[F+(s+2) F^{\prime \prime}\right]\right\}=0 .
\end{aligned}
$$


Noticing $F^{2}+F^{2} \neq 0,(3.12)$ can be divided into two equations:

$$
\begin{aligned}
& F^{\prime}=0 \\
& (s-1)\left(F+F^{\prime \prime}\right)\left[F^{2}+(s+2) F^{\prime 2}\right]+2\left(F^{2}+F^{\prime 2}\right)\left[F+(s+2) F^{\prime \prime}\right]=0 .
\end{aligned}
$$

The solution for case $p_{1}=\mathrm{p}_{2}$ should satisfy one equation among (3.10), (3.13) and (3.14). The general solution of $(3.10)$ is

$$
F(\theta)=A \cos \theta+B \sin \theta
$$

where $A$ and $B$ are constants to be determined. Accordingly, (3.6), (3.1) and (3.5) become

$$
\begin{aligned}
& w / 2 \varepsilon_{R}=w_{0}+r(\mathrm{~A} \cos \theta+B \sin \theta) \\
& \left.\begin{array}{l}
\left.\left\{\begin{array}{l}
\tau_{x} \\
\tau_{y}
\end{array}\right\}=\mu(1-D)\left\{\begin{array}{l}
\gamma_{x} \\
\gamma_{y}
\end{array}\right\}=\mu(1-D) 2 \varepsilon_{R}\left\{\begin{array}{l}
A \\
B
\end{array}\right\}\right\} \\
D=\left(\varepsilon_{e q} \varepsilon_{R}\right)^{s+1}=\left(\sqrt{A^{2}+B^{2}}\right)^{s+1}=\text { const. }
\end{array}\right\} .
\end{aligned}
$$

The domain described by (3.16) is called constant damage zone.

Obviously, $F(\theta)=$ const. is the solution of (3.13). The solution should also satisfy (3.11) which is the equation before differentiation. Then $F(\theta)=1$ and $D(\theta)=1$, i.e. the material is fully damaged. Hereafter we shall treat the fully damaged zone as a void in which the stresses are zero everywhere, the displacement and strains can not be described.

To solve (3.14) the transform is introduced

$$
\left.\begin{array}{l}
F(\theta)=X(\theta) \cos Y(\theta) \\
F^{\prime}(\theta)=X(\theta) \sin Y(\theta)
\end{array}\right\}
$$

Then (3.17) and (3.14) lead to

$$
\begin{aligned}
& X^{\prime}(\theta)=X(\theta)\left[1+Y^{\prime}(\theta)\right] \tan Y(\theta) \\
& Y^{\prime}(\theta)=-\left[1+(s+1) \sin ^{2} Y(\theta)\right] /\left[3+(s-1) \sin ^{2} Y(\theta)\right] .
\end{aligned}
$$

Under the boundary conditions $X(\theta)=X_{0}, Y(\theta)=Y_{0}$, at $\theta=\theta_{0}$, the solutions of (3.19) and (3.18) are

$$
\begin{aligned}
& \theta-\theta_{0}+\frac{s-1}{s+1}\left[Y(\theta)-Y_{0}\right]+\frac{2 \sqrt{s+2}}{s+1}\left[\tan ^{-1}(\sqrt{s+2} \tan Y(\theta))-\right. \\
& \left.-\tan ^{-1}\left(\sqrt{s+2} \tan Y_{0}\right)\right]=0 \\
& X(\theta)=X_{0}\left\{\left[1+(s+1) \sin ^{2} Y_{0}\right] /\left[1+(s+1) \sin ^{2} Y(\theta)\right]\right\}^{1 /(s+1)} .
\end{aligned}
$$

As before, satisfaction of $(3.11)$ requires

$$
X_{0}\left[1+(s+1) \sin ^{2} Y_{0}\right]^{1 /(s+1)}=1 .
$$


From (3.21) and (3.22) we have

$$
X(\theta)=\left[1+(s+1) \sin ^{2} Y(\theta)\right]^{-1 /(s+1)}
$$

Substituting (3.23) and (3.17) into (3.6), (3.3), (3.1) and (3.7), we have

$$
\left.\begin{array}{l}
w / 2 \varepsilon_{R}=w_{0}+r X(\theta) \cos Y(\theta) \\
\left.\left\{\begin{array}{l}
\tau_{\nu} \\
\tau_{\theta}
\end{array}\right\}=\mu(1-D)\left\{\begin{array}{l}
\gamma_{\gamma} \\
\gamma_{\theta}
\end{array}\right\}=\mu(1-D) 2 \varepsilon_{R} X(\theta)\left\{\begin{array}{l}
\cos Y(\theta) \\
\sin Y(\theta)
\end{array}\right\}\right\} . \\
D(\theta)=[X(\theta)]^{s+1}=\left[1+(s+1) \sin ^{2} Y(\theta)\right]^{-1}
\end{array}\right\} .
$$

From the last equation in (3.24) we know

$$
D^{*} \leqslant D \leqslant 1 \text {. }
$$

Therefore the domain described by (3.24) is called the softening zone. In summary the case $p_{1}=p_{2}$ has two solutions, i.e. (3.16) and (3.24).

\subsection{Case $p_{1}>p_{2}$}

In (3.8) the damage term dominates, $q<1$ from (3.9). Because the strains must be bounded in both hardening zone and softening zone, the condition $q<1$ which leads to the singularity of strain is in contradiction to the constitutive law. Consequently the case $p_{1}>\mathrm{p}_{2}$ must be abandoned.

\subsection{Case $p_{1}<p_{2}$}

In (3.8) the elasticity term dominates. From (3.9) $q>1$. The basic equation (3.8) reduces to

$$
q^{2} F(\theta)+F^{\prime \prime}(\theta)=0
$$

whose general solution is

$$
F(\theta)=A \cos (q \theta)+B \sin (q \theta)
$$

In the domain controlled by (3.26), we have

$$
\begin{aligned}
& w / 2 \varepsilon_{R}=w_{0}+r^{q}[\mathrm{~A} \cos (q \theta)+B \sin (q \theta)] \\
& \left.\begin{array}{rl}
D= & r^{(q-1)(s+1)}\left[q\left(A^{2}+B^{2}\right)^{1 / 2}\right]^{s+1} \\
\left\{\begin{array}{l}
\tau_{r} \\
\tau_{\theta}
\end{array}\right\} & =\mu(1-D)\left\{\begin{array}{l}
\gamma_{r} \\
\gamma_{\theta}
\end{array}\right\}= \\
& =\mu(1-D) 2 \varepsilon_{R} q r^{(q-1)}\left\{\begin{array}{l}
A \cos (q \theta)+B \sin (q \theta) \\
-A \sin (q \theta)+B \cos (q \theta)
\end{array}\right\}
\end{array}\right\}
\end{aligned}
$$


Because $q>1$ and $s+1>0,(3.28)$ describes a hardening zone wherein the strains, stresses and damage tend to zero as $r \rightarrow 0$.

\section{Construction of the crack-tip field}

Denote the boundary line between any two domains by $\Gamma$ and the discontinuity of quantity $\psi$ across $\Gamma$ by $[\psi]_{\Gamma}$. For anti-plane shear problem the basic contiguity conditions are

$$
[w]_{\Gamma}=\left[\tau_{\theta}\right]_{\Gamma}=0
$$

By use of (3.6) and (3.1), the above two conditions can be expressed as follows

$$
\begin{aligned}
& {[F]_{\Gamma}=0,} \\
& {\left[(1-D) \gamma_{\theta}\right]_{\Gamma}=0 .}
\end{aligned}
$$

Equation (4.2) can be satisfied in two manners

(a) $\left[\gamma_{\theta}\right]_{\Gamma}=[D]_{\Gamma}=0$, i.e. $\left[F^{\prime}\right]_{\Gamma}=0$,

(b) $\left[\gamma_{\theta}\right]_{\Gamma} \neq 0,[D]_{\Gamma} \neq 0$, i.e. $\left[F^{\prime}\right]_{\Gamma} \neq 0$.

The traction-free condition $\tau_{\theta}=0$ should be satisfied at the crack surface and at the boundary line neighboring with the fully damaged zone (i.e. void).

Due to the difference of the exponent $q$, it is obvious that the hardening zone $(q>1)$ and the softening zone ( $q=1$ ) can not be neighbours to each other.

In constructing the crack-tip field, we give priority to the scheme shown in Fig. 2(a). In the scheme the fully damaged zone (D) is ahead of the crack, zones (S) and (C) are described by (3.24) $\left(D^{*} \leqslant D \leqslant 1\right)$ and (3.16) $(D=$ const.), respectively. From the condition on the crack surface, the coefficient $B=0$ in (3.16). At $\Gamma_{0}$ the contiguity condition is

$$
D=1, \text { at } \theta=\theta_{0}
$$

Using (3.24) $)_{4}$, the above formula leads to

$$
\sin Y\left(\theta_{0}\right) \equiv \sin Y_{0}=0 \quad \text { or } \quad Y_{0}=n \pi, n=0, \pm 1, \pm 2, \cdots
$$

Without loss of generality, take $n=0$. Thus, (3.20) can be reduced to

$$
\theta-\theta_{0}+\frac{s-1}{s+1} Y(\theta)+\frac{2 \sqrt{s+2}}{s+1} \tan ^{-1}[\sqrt{s+2} \tan Y(\theta)]=0
$$

At $\Gamma_{1}$ the contiguity conditions (4.1) and (4.2) respectively become

$$
\begin{aligned}
& A \cos \theta_{1}=X_{1} \cos Y_{1}, \\
& -A\left(1-A^{s+1}\right) \sin \theta_{1}=\left(1-X_{1}^{s+1}\right) X_{1} \sin Y_{1} .
\end{aligned}
$$




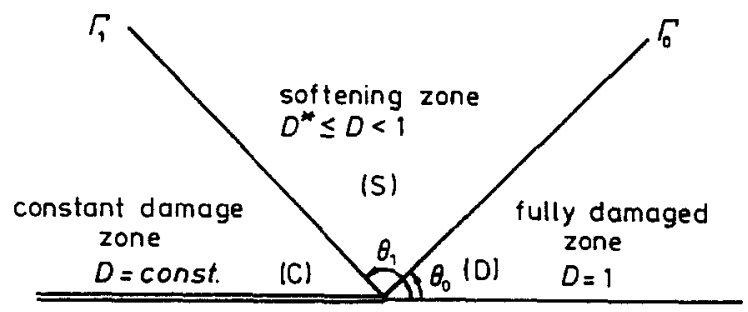

2 (a)

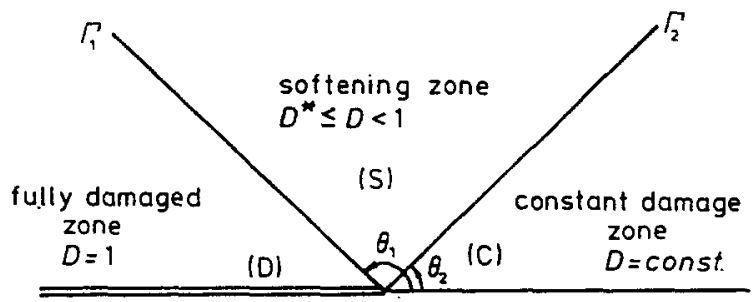

$2(b)$

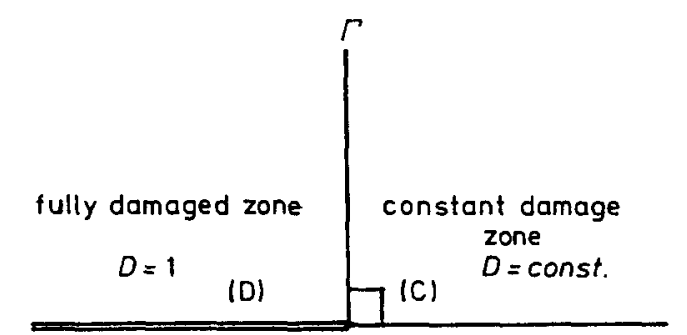

$2(c)$

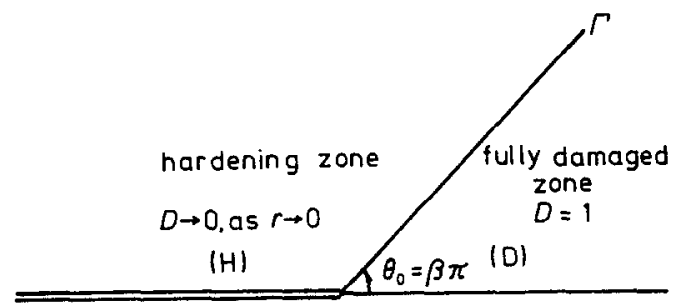

2 (d)

Fig. 2. Schemes of constructing crack-tip field.

Denoting $Y\left(\theta_{1}\right)$ by $Y_{1}$, from (3.23) we have

$$
X_{1} \equiv X\left(\theta_{1}\right)=\left[1+(s+1) \sin ^{2} Y_{1}\right]^{-1 /(s+1)} .
$$

Letting $\theta=\theta_{1}$ in $(3.20)^{\prime}$, we have

$$
\theta_{1}-\theta_{0}+\frac{s-1}{s+1} Y_{1}+\frac{2 \sqrt{s+2}}{s+1} \tan ^{-1}\left(\sqrt{s+2} \tan Y_{1}\right)=0
$$


Eliminating $A$ from (4.3) and (4.4), we obtain a relation between $\theta_{1}$ and $Y_{1}$. Associating the relation with (3.20)", the values of $\theta_{1}$ and $Y_{1}$ can be determined for assigned $s$ and assumed $\theta_{0}$. The above mentioned process has been done for $s=2,7,14,23,34,47,62,79$, and 98 . The shooting $\theta_{0}$ values are taken in the interval $[0, \pi]$ in increments of 0.1 . Disappointingly, no $\theta_{1}$ is found which satisfies $\theta_{0} \leqslant \theta_{1} \leqslant \pi$. Therefore the solution does not exist for the scheme in Fig. 2(a). After examination of all possible schemes of the crack-tip field with the upper half plane composed of no more than three domains which could be softening, constant damage or fully damaged zones, we found that there exist only two solutions corresponding to the schemes shown in Fig. 2(b) and 2(c).

In the scheme in Fig. 2(b), (3.16) describes domain (C). The antisymmetric condition leads to $w_{0}=A=0$ in (3.16). So (3.16) becomes

$$
\left.\begin{array}{l}
w / 2 \varepsilon_{R}=B r \sin \theta \\
\tau_{x}=0 \\
\tau_{y}=\mu(1-D) \gamma_{y}=\mu(1-D) 2 \varepsilon_{R} B \\
D=B^{s+1}
\end{array}\right\}, \quad 0 \leqslant \theta \leqslant \theta_{2} .
$$

Domain (D) is the fully damaged zone. Domain (S) is the softening zone represented by (3.24), where $D^{*}=D_{\min } \leqslant D \leqslant 1$. Thus the amplitude factor $B$ of the crack-tip field should meet $B_{\min } \leqslant B \leqslant 1$ from the contiguity condition at $\Gamma_{2}$. The value of $B_{\min }$ depends on $s$

$$
B_{\min }=D_{\min }^{1 /(s+1)}=(s+2)^{-1 /(s+1)} \text {. }
$$

Introducing a normalized amplitude factor $\bar{B}=\left(B-B_{\min }\right) /\left(1-B_{\min }\right)$, we always have $0 \leqslant \bar{B} \leqslant 1$ for any $s$. The curves of $\theta_{1}$ and $\theta_{2}$ against $\bar{B}$ are depicted in Fig. 3. For $s=2$ the

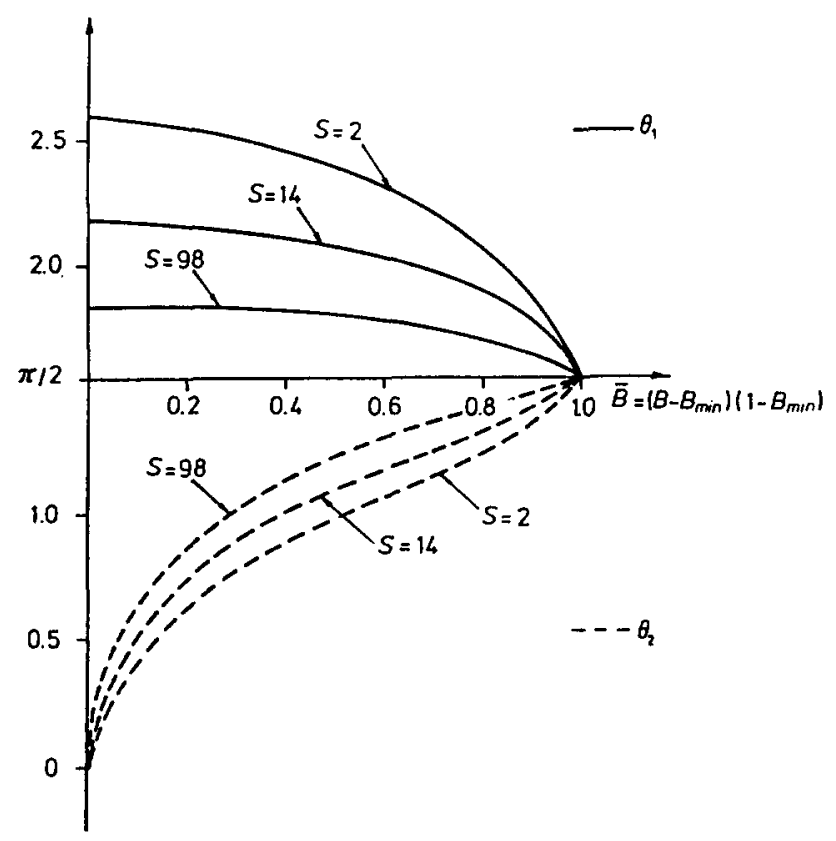

Fig. 3. Variation of angles $\theta_{1}$ and $\theta_{2}$ with $\bar{B}$ in scheme $2(b)$. 


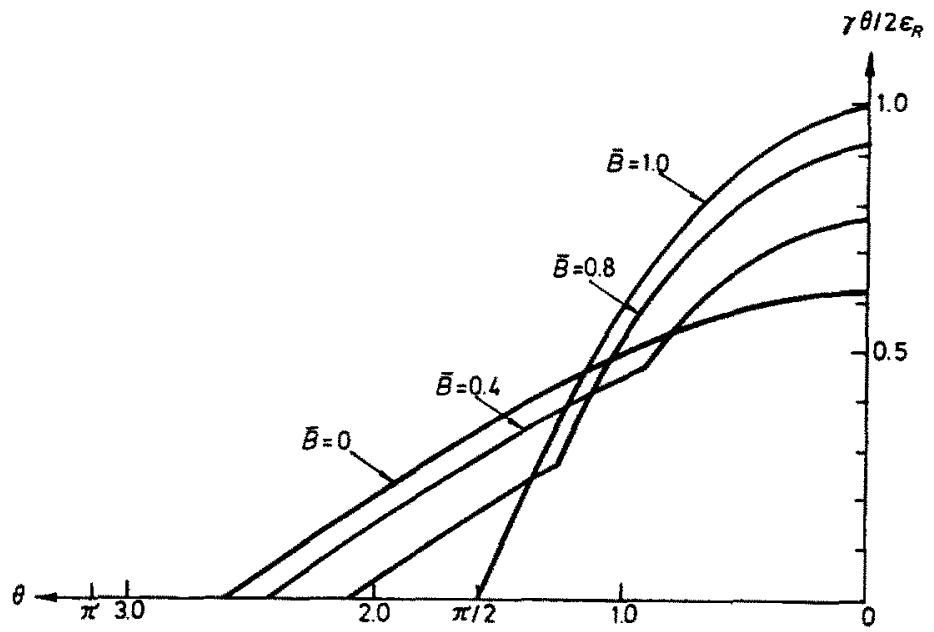

Fig. 4. $\theta$-distributions of $\gamma_{\theta} / 2 \varepsilon_{R}$ for $S=2$.

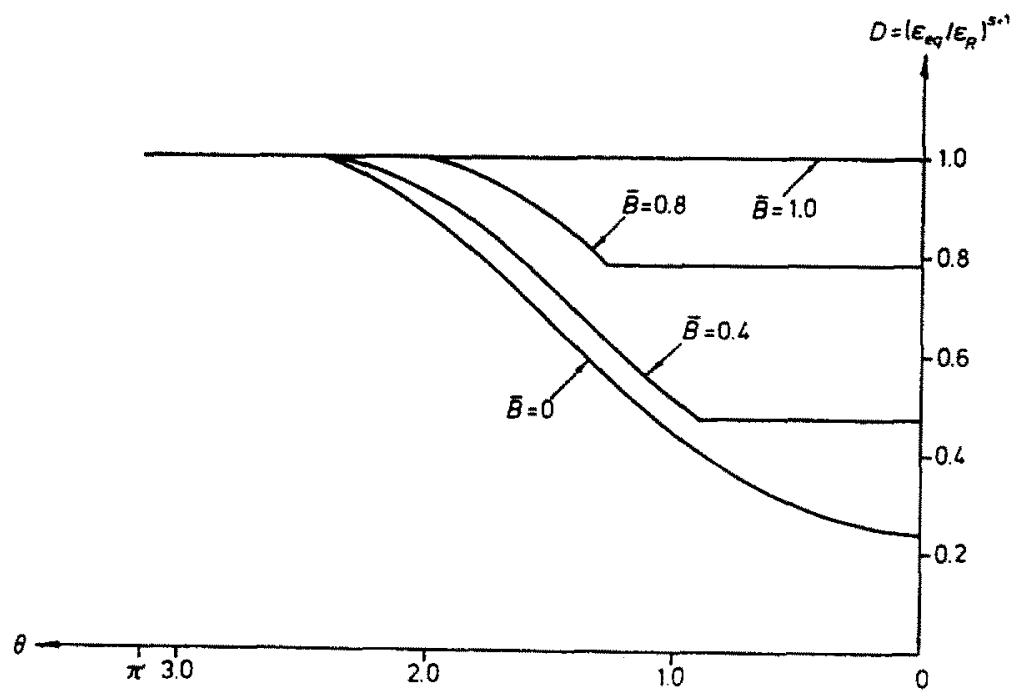

Fig. 5. $\theta$-distributions of $D=\left(\varepsilon_{e q} / \varepsilon_{R}\right)^{s+1}$ for $S=2$.

angular distribution curves of $\gamma_{\theta}, D=\left(\varepsilon_{e q} / \varepsilon_{R}\right)^{s+1}$ and $\tau_{e q}$ are pictured in Figs. 4, 5, 6. The curves indicate that $\Gamma_{2}$ is a weak discontinuity line. Figure 5 shows that the active loading condition $\mathrm{d} \varepsilon_{e q} \geqslant 0$ is satisfied everywhere as $B$ increases.

In the scheme shown in Fig. 2(c), the boundary line $\Gamma$ is perpendicular to the crack-plane. The fully damaged zone (D) is situated by the flank of the crack and constant damage domain (C) is ahead of the crack. As in the scheme in Fig. 2(b), the displacement, stresses and damage are represented by $(4.5)$ in zone $(C)$. Constant damage zone $(C)$ of the scheme 2(c) differs from that of the scheme $2(\mathrm{~b})$ in that the constant damage value $D$ can vary in range $(0,1)$. Depending on value of $D$, zone $(C)$ can be hardening or softening zone in the scheme $2(\mathrm{c})$. In both schemes, Fig. 2(b) and Fig. 2(c), fully damaged zone is situated by the flank of the crack. About this point discussion will be given in the next section.

The last scheme is shown in Fig. 2(d) which corresponds to $q>1$, i.e. case $p_{1}<p_{2}$. The hardening zone $(\mathrm{H})$ is represented by $(3.28)$. The fully damaged zone is located ahead of the 


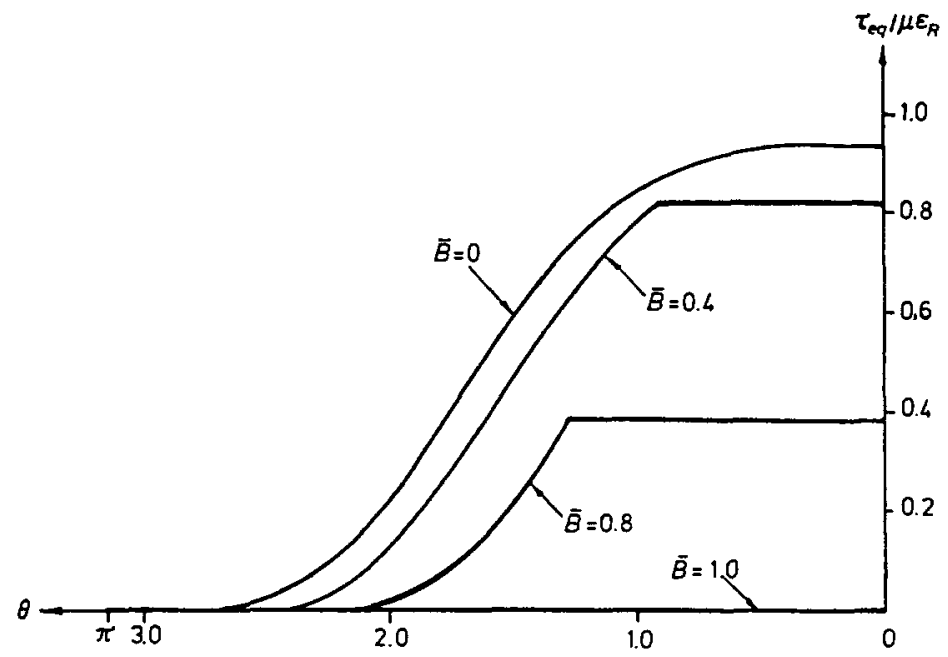

Fig. 6. $\theta$-distributions of $\tau_{e q} / \mu \varepsilon_{R}$ for $S=2$.

crack. The conditions $\tau_{\theta}=0$ at $\theta=\theta_{0}$ and $\pi$ can be written

$$
\left.\begin{array}{l}
-\sin \left(q \theta_{0}\right) A+\cos \left(q \theta_{0}\right) B=0 \\
-\sin (q \pi) A+\cos (q \pi) B=0
\end{array}\right\}
$$

Nontrivial solution requires

$$
\left|\begin{array}{ll}
-\sin \left(q \theta_{0}\right) & \cos \left(q \theta_{0}\right) \\
-\sin (q \pi) & \cos (q \pi)
\end{array}\right|=0
$$

\section{Hence}

$$
q\left(\pi-\theta_{0}\right)=n \pi, \quad n=0, \pm 1, \pm 2, \cdots .
$$

Due to $q>1, n$ must be positive. Considering only the leading term of the asymptotic solution, we should take $n=1$, that is

$$
q=\pi /\left(\pi-\theta_{0}\right) \equiv 1 /(1-\beta), \quad \beta=\theta_{0} / \pi
$$

From (4.6), we have

$$
B / A=\tan \left(\frac{\pi}{1-\beta}\right) .
$$

Whenever the fully damaged zone angle $\theta_{0}$ (or $\beta$ value) is obtained, the singularity exponent $q$ and the angular distribution functions in (3.28) can be determined. 


\section{Discussion and conclusion}

In Section 4 three possible solutions of the crack-tip field are found, i.e. schemes shown in Figs. 2(b), 2(c) and 2(d), which totally satisfy the basic equation and all required conditions. Therefore they are all mathematically reasonable. However except for the scheme in 2(d), in both schemes 2(b) and 2(c) the fully damaged zones (or, say, the voids) are behind the crack tip. The actual observations show that the void or the most seriously damaged zone appears ahead of the crack tip generally, although the major observations proceed in the condition of mode-I. It is for this reason, that the schemes which were obtained four years ago have not been submitted for publication till now. Recently, the authors obtained a closed solution [5] of mode-III problem for an elasto-brittle damaged material (equivalently $s \rightarrow \infty$ in (2.5)) in which the schemes 2(b) and 2(c) (with $s \rightarrow \infty$ ) can be implemented as the local field around the frontier of the fully damaged zone situated ahead of the crack tip.

First, let us examine the scheme in Fig. 2(b) in which the softening zone (S) is described by (3.20). Letting $s \rightarrow \infty$ and taking $\theta_{0}=\theta_{1}, Y_{0}=Y_{1}=0$ at $\Gamma_{1},(3.20)$ reduces to

$$
Y(\theta)=\theta_{1}-\theta,
$$

while (3.23) gives $X(\theta) \rightarrow 1$ and (3.24) leads to $D(\theta) \rightarrow 0$ in domain (S). The contiguity conditions at $\Gamma_{2}$ become

$$
\left.\begin{array}{l}
\cos \left[Y\left(\theta_{2}\right)\right]=B \sin \theta_{2} \\
\sin \left[Y\left(\theta_{2}\right)\right]=B \cos \theta_{2}
\end{array}\right\}
$$

Combining (5.1) and (5.2) we have $\theta_{1} \rightarrow \frac{1}{2} \pi$ and $B \rightarrow 1$ while $\theta_{2}\left(0<\theta_{2}<\theta_{1}\right)$ is undetermined. From (4.5) $)_{4} D=B^{S+1} \rightarrow 0$ in domain (C). Then zones (C) and (S) coalesce to an undamaged elastic zone, so the angle $\theta_{2}$ is of no significance. The crack-tip fields in both (C) and (S) become (from either (4.5) or (3.24))

$$
\left.\begin{array}{l}
w \rightarrow 2 \varepsilon_{R} r \sin \theta \\
\tau_{x}=0 \\
\tau_{y} \rightarrow 2 \varepsilon_{R} \mu \\
D \rightarrow 0
\end{array}\right\}
$$

Thus the scheme in Fig. 2(b) coincides with the local field around the frontier of the fully damaged zone in the HZH solution (see (4.6) ${ }_{B}$ and (4.5) $)_{B}$ in [5]), as shown in Fig. 7.

As for the scheme 2(c), it is obvious that the fields of domain (C) are described by (5.3) under the conditions of $s \rightarrow \infty, B \rightarrow 1$ and $B^{S+1} \rightarrow 0$ as well. And so the relation between the scheme 2(c) and $\mathrm{HZH}$ solution an be displayed on Fig. 7, too.

Finally, we would c . .pare the scheme 2(d) with [5]. Substitute (4.7) into (3.28) and write the Cartesian component, of stress

$$
\begin{aligned}
& w / 2 \varepsilon_{R}=w_{0}+A_{0} r^{1 /(1-\beta) \cos [(\pi-\theta) /(1-\beta)]} \\
& \left.\begin{array}{l}
D=r^{(s+1) \beta /(1-\beta)}\left[\sqrt{A^{2}+B^{2}} /(1-\beta)\right]^{s+1} \\
\left\{\begin{array}{l}
\tau_{x} \\
\tau_{y}
\end{array}\right\}=-A_{1} r^{\beta /(1-\beta)}\left\{\begin{array}{l}
\cos [(\pi-\theta) \beta /(1-\beta)] \\
\sin [(\pi-\theta) \beta /(1-\beta)]
\end{array}\right\}
\end{array}\right\}, \text { for any value of } s
\end{aligned}
$$

where $A_{0}=A \sec [\pi /(1-\beta)], A_{1}=A_{0}\left[2 \varepsilon_{R} \mu /(1-\beta)\right]$. 


\section{schemes 2(b) and 2(c) with $s \rightarrow \infty$}

can be regarded as the local solution

contour of fully damaged zone

around frontier of fully damaged zone

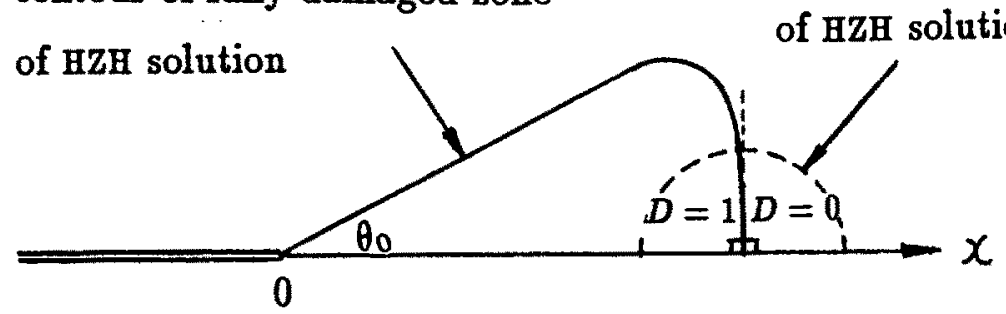

Fig. 7. Relation between schemes 2(b), 2(c) and HZH solution [5].

From $(5.4)_{2} D \rightarrow 0$ as $s \rightarrow \infty$ and $r \rightarrow 0$. The forms of $(5.4)_{1}$ and $(5.4)_{3}$ coincide with those of the asymptotic expansions of the HZH solution at the crack tip (see (4.6) and (4.5) in [5]). Guo et al. [6] obtained the finite element solution for the material of $s \rightarrow \infty$ in which the angle $\theta_{0}$ subtended by the fully damaged zone is above $42.4^{\circ}$, corresponding to $q \approx 1.31$ in (4.7). It is reasonable to expect that the angle $\theta_{0}$, consequently $q$, will depend on the material constant $s$.

As discussed above, the validity of scheme 2(d) is undoubted both mathematically and physically. It is a fly in the ointment that up to now $\theta_{0}$ and $q$ should be determined by finite element method. By means of comparing with [5] we have sought an acceptable physical explanation for the schemes 2(b) and 2(c), that is, they may be the local solutions around the frontier of the fully damaged zone. Based on the explanation we can expect that for any value of $s \neq \infty$ the frontier of the fully damaged zone which is ahead of the crack tip may be inclined or perpendicular to the $x$-axis, respectively, corresponding to the scheme 2(b) and 2(c), as shown in Fig. 2(b) and 2(c). Perhaps, gentle readers would ask whether the schemes 2(b) and 2(c) with fully damaged zone behind the crack tip can be directly regarded as the crack-tip fields. That remains to be examined by the analytical full field solution or the experiments of mode-III.

In principle, the constitutive model and the method of attack in the present paper can be extended to the mode-I problem, but with much more mathematical difficulties.

\section{Acknowledgement}

The project was supported by the National Science Foundation of China.

\section{References}

1. J. Lemaitre and J.-L. Chaboche, Mécanique des Materiaux Solides, Chapitre 7, \$5.1, Dunold (1985).

2. H.D. Bui and A. Ehrlacher, Advances in Fracture Research. Proceedings of the Fifth International Conference on Fracture, D. Francois (ed.) 2 Pergamon Press (1981) 533-551.

3. C.L. Chow and T.J. Lu, International Joumal of Fracture 50 (1991) 79-114.

4. J. Wang and C.L. Chow, International Journal of Fracture 54 (1992) 165-183.

5. T.H. Hao, X.T. Zhang and K.C. Hwang, Acta Mechanica Sinica (China) 7 (1991) 351-359.

6. T.F. Guo, K.C. Hwang and X.T. Zhang, in Computational Methods in Engineering-Aduances and Application, Vol. 2 , A.A.O. Tay and K.Y. Lam (eds.), World Scientific (1992). 